\title{
Evaluation of the respiratory system of non-alcohol drug addicted patients
}

\author{
Fulya Çiyiltepe ${ }^{1}$, Hüseyin Çiyiltepe ${ }^{2}$, Hatice Uluçoban Dede ${ }^{3}$, Aliye Candan Öğüş ${ }^{3}$
}

\author{
${ }^{1}$ Department of Intensive Care Unit, Haydarpasa Education and Training Hospital, \\ Istanbul, Turkey \\ 2Department of Gastroenterological Surgery, Kartal Kosuyolu High Speciality \\ Education and Training Hospital, Istanbul, Turkey \\ ${ }^{3}$ Department of Pulmonary Medicine, School of Medicine, Akdeniz University, Antalya, \\ Turkey
}

Submitted: 12 January 2017

Accepted: 7 February 2017

Arch Med Sci Civil Dis 2017; 2: e21-e26

DOI: https://doi.org/10.5114/amscd.2017.66358

Copyright @ 2017 Termedia \& Banach

\begin{abstract}
Introduction: One of the diseases that has left a mark on our age is psychoactive substance addiction. The onset of narcotic substance abuse has dropped to very early ages of life in our country. The prevalence of abuse of such substances as heroin and cocaine through inhaling is increasing dramatically. Abusing these substances by inhalation has life-threatening risks such as bronchospasm, alveolar hemorrhage and acute respiratory syndrome. Our aim in this study was to describe possible respiratory system risks in individuals who had substance addiction.

Material and methods: The study was conducted with 40 healthy and 40 drug addicted people who receive treatment at AMATEM department in Akdeniz University Medicine Faculty. The data were interpreted according to the results of the anamnesis, physical examination, chest $X$-ray, spirometry and respiratory questionnaire for both test groups, and the final evaluation was carried out using these data.

Results: For the addicted group, the symptoms related to the respiratory system and the symptom scores of the respiratory questionnaire were found to be significantly high during our study $(p<0.05)$.

Conclusions: Consequently, the results of our study revealed that substance addicts most frequently preferred the inhalation mode when abusing narcotic substances. Moreover, it was also determined that they were heavily smoking besides narcotic substance abuse. We believe that efforts to prevent smoking addiction in society can be developed so as to include narcotic substance abuse, especially among young individuals, which in turn can contribute to a decrease in future risks.
\end{abstract}

Key words: heroin, cocaine, respiratory system.

\section{Introduction}

One of the diseases that has left a mark on our age is psychoactive substance addiction. Although psychoactive substances have been used by many societies at varying degrees for good or ill reasons for many years, the practice has not been as effective as today. The discovery of different, more efficient, and harder-to-treat new substances, common abuse of these substances by a larger portion of societies, especially by young people who form the dynamic structure of societies, and their

\author{
Corresponding author: \\ Fulya Çiyiltepe \\ Department of \\ Intensive Care Unit \\ Haydarpasa Education \\ and Training Hospital \\ 34000 Istanbul, Turkey \\ E-mail: drciyiltepe@hotmail. \\ com
}


jeopardizing their futures can be listed as the underlying reasons for this effect $[1,2]$.

The fact that the onset of narcotic substance abuse has dropped to very early ages of life in our country, as is the case all over the world, has led to a prolongation of the period in which individuals are exposed to such substances [1, 3, 4]. Individuals have started to develop a tendency to use other means of narcotic substance abuse, and there has especially been a dramatic increase in the prevalence of abuse of such substances as heroin and cocaine through inhaling, subsequent to the widespread abuse of such substances via different means and because of the increase in awareness of contagious diseases spreading through common injector use $[5,6]$. Substance abusers, however, have not yet developed awareness about the effects of these inhaled substances on the respiratory system in the short and long terms. Despite the fact that there is only a limited number of studies on this subject in the literature, there are also studies which have reported that individuals who abused narcotic substances through inhaling risked serious life-threatening clinical pictures varying from bronchospasm to alveolar hemorrhage and acute respiratory syndrome $[6,7]$.

Our aim in this study was to describe possible respiratory system risks by contributing to unearthing respiratory problems in individuals who had substance addiction other than alcohol and presented to the Alcohol and Substance Addiction Research and Training Center by using a screening method.

\section{Material and methods}

While the study group was formed by individuals who had been hospitalized at Akdeniz University Medical School's Alcohol and Substance Addiction Research and Training Center to be treated for substance addiction other than alcohol between April 2013 and October 2013, the control group was formed by healthy young individuals who had presented to our outpatient clinic in order to obtain a Medical Board Report to be handed for enrollment at the Academy of Physical Education and the Academy of Health Sciences.

While the study and control groups were formed, all individuals conforming to the criteria of our study were informed about the study and those who agreed to participate in the study were included following the acquisition of their written consent. Patients in the study group were selected based on the order they had been hospitalized regardless of their age, sex, history of smoking, type of substance abused, and the mode of abuse. Patients' and healthy participants' demographic data, anamneses, physical examination results, and history of smoking were recorded. The spi- rometric measurement test and St. George's Respiratory Questionnaire (SGRQ) were conducted and evaluated on the same day. All participants' PA pulmonary radiography results were evaluated.

Akdeniz University Medical School's Board of Ethics approved our study, and the study was conducted in line with the principles laid out by the Declaration of Helsinki. Moreover, our study was supported by Akdeniz University's Scientific Research Projects Coordination Unit (Project no: 2013.04.0103.017). Data collected within the scope of the study were analyzed by PASW 18 (SPSS/IBM, Chicago, IL, USA).

The participants' information on their ages, sexes, professions, and places of residence was recorded. Whether the participants had dyspnea, coughing, phlegm, hemoptysis, chest pain, and bronchial hyperresponsiveness symptoms was questioned respectively. The spirometric measurements were conducted by an experienced respiratory technician in accordance with the requirements of the method used at our clinic's pulmonary function test (PFT) laboratory by using a Jaeger Lab Manager V5.50.0 pulmonary function test tool $[8,9]$.

All participants' PA pulmonary radiography results were evaluated. Pathologies of the parenchyma, mediastinum, and soft tissue were interpreted within the scope of radiography evaluation.

The patients were given oral information about the Turkish version of the 72-question SGRQ, adapted to Turkish by Prof. Dr. Türkan Tatlıcıoğlu. The questionnaire was conducted at the outpatient clinic by reading the questions to the participants, and it was scored depending on the responses, while the questions that the participants could not understand fully were explained. The parts of the questionnaire where the efficiency of chronic obstructive pulmonary disease (COPD) treatment was questioned were scored without having them applied to any participant [10].

\section{Statistical analysis}

Descriptive statistics such as frequency distribution, mean figures, and standard deviation were used to describe sampling. The hypothesis for the distribution of normality was investigated by the Shapiro-Wilk test. While the differences among the mean figures of two independent groups were explored by Student's $t$-test in cases where the parametric test hypotheses were achieved, the non-parametric alternatives of these tests, namely, the Mann-Whitney $U$ test and the Kruskal-Wallis test, were used for two groups and for more than two groups respectively in cases where parametric test hypotheses were not obtained. Categorical data were evaluated by the $\chi^{2}$ significance test or Fisher's exact test. In order to pinpoint the differences the $95 \%$ significance level (or $\alpha=0.05$ 
error rate) was used within the framework of the conducted analyses.

\section{Results}

The group covering substance addicts was classified as group I, while the control group was classified as group II. The patient group had 40 participants and the control group had 40 participants in our study. All participants were male.

The mean age of substance addicts was significantly higher than that of the control group. The youngest age in both groups was 17 and the mean age of group I was $25 \pm 1$, while it was $18 \pm 1$ for group II. There was no statistically significant difference between the groups regarding body mass index (BMI).

The presence of five main respiratory symptoms, which were obtained through detailed anamneses of all the participants covered by the study - shortness of breath, coughing, phlegm, chest pain, and hemoptysis - was significantly higher in group I (Table I).

History of smoking and duration of exposure, asbestos exposure, bronchial hyperresponsivity, co-morbid diseases, bronchodilator use, and features of personal and familial histories of the participants in both groups were compared. In the substance abuse group the patients were all smokers and their rates of smoking and duration of smoking were significantly higher than those of the control group. The starting age of smoking in group I was found to be statistically significantly lower than the control group (13 years and 18 years of age respectively, $p<0.001)$.

When the participants' vital signs, respiratory system auscultation, presence of digital clubbing, and pulmonary PA results were compared between the groups, it was found that parameters such as respiratory rate (RR), heart rate (HR), rhonchus, and the presence of pathology were significantly higher and oxygen saturation $\left(\mathrm{SpO}_{2}\right)$ was significantly lower in group I.

When the maximum, mean, and minimum spirometry results of groups I and II were compared in both liters and percentages, it was determined that forced expiratory volume in $1 \mathrm{~s}(\mathrm{FEV}) /$ forced vital capacity (FVC) levels in group I were significantly lower than those of the control group. When the SGRQ questionnaire was evaluated, the same parts were taken into consideration for both groups and the scoring was performed accordingly. It was observed that each symptom that was questioned in group I was significantly higher than group II (Table II).

All 40 individuals making up group I had been heroin abusers (100\%). Of these individuals, 12 (30\%) had been abusing heroin together with cocaine, 11 (27.5\%) had been abusing heroin together with marijuana, and 17 (42.5\%) had been abusing only heroin. Twenty-five $(62.5 \%)$ out of 40 , who had been abusing heroin, had preferred the inhaler mode, 2 (5\%) had preferred the IV mode, and 13 (32.5\%) had used both the inhaler and IV modes. The most common mode of abuse was the inhaler mode, with 7 (17.5\%) individuals within the group who had abused heroin together with cocaine. Two (5\%) individuals had preferred both the IV and inhaler modes, while 3 (7.5\%) individuals had only preferred the IV mode. It was determined that the inhaler mode was significantly more common than the IV mode of substance abuse when the modes of substance abuse were compared.

\section{Discussion}

Substance abuse and addiction are significant public health problems in our country, as they are in the world, and they yield many social results. The United Nations Office on Drugs and Crime (UNODC) reported that 208 million individuals in the 15-64 years age group had abused at least one substance within the previous year, and this figure equals $4.8 \%$ of the population within the same age range. In other words, the report stated that approximately one individual in every twenty within the 15-64 age group abused a substance at least once within the previous year [1, 2].

The first questionnaire, which was conducted in 2002 with 7681 individuals on the prevalence of substance abuse in our country, reported that the rate of addictive substance abuse other than

Table I. Respiratory symptoms

\begin{tabular}{|lccccc|}
\hline Symptom & \multicolumn{2}{c}{ Group 1 } & \multicolumn{2}{c}{ Group 2 } & \multirow{2}{*}{-value } \\
\cline { 2 - 5 } & $n$ & $\%$ & $n$ & $\%$ & \\
\hline Shortness of breath & 28 & 70 & 0 & 0 & $<0.001^{*}$ \\
\hline Cough & 20 & 50 & 0 & 0 & $<0.001^{*}$ \\
\hline Phlegm & 19 & 47.5 & 2 & 5 & $<0.001^{*}$ \\
\hline Chest pain & 13 & 32.5 & 0 & 0 & $<0.001^{*}$ \\
\hline Hemoptysis & 9 & 22.5 & 0 & 0 & $<0.001^{*}$ \\
\hline *Statistically significant & & & & &
\end{tabular}

${ }^{\star}$ Statistically significant 
Table II. Spirometry and SGRQ results

\begin{tabular}{|c|c|c|c|c|c|}
\hline Parameter & Group & Minimum & Maximum & Mean & $P$-value \\
\hline \multirow[t]{2}{*}{$\mathrm{FEV}_{1}(\mathrm{It})$} & 1 & 2.5 & 5.7 & 4.2 & 0.152 \\
\hline & 2 & 3.4 & 5.1 & 4.4 & \\
\hline \multirow[t]{2}{*}{$\mathrm{FEV}_{1}(\%)$} & 1 & 60 & 123 & 98 & 0.233 \\
\hline & 2 & 84 & 119 & 101 & \\
\hline \multirow[t]{2}{*}{ FVC (lt) } & 1 & 3.4 & 6.3 & 4.7 & 0.502 \\
\hline & 2 & 3.3 & 6.2 & 4.7 & \\
\hline \multirow[t]{2}{*}{ FVC (\%) } & 1 & 62 & 120 & 92 & 0.287 \\
\hline & 2 & 71 & 109 & 89 & \\
\hline \multirow[t]{2}{*}{$\mathrm{FEV}_{1} / \mathrm{FVC}$} & 1 & 65 & 99 & 86 & $<0.001^{*}$ \\
\hline & 2 & 77 & 99 & 94 & \\
\hline \multirow[t]{2}{*}{ SGRQ } & 1 & 0 & - & 5 & 0.009 \\
\hline & 2 & 0 & 12 & 2 & \\
\hline
\end{tabular}

*Statistically significant.

alcohol and tobacco was $0.3 \%$ and the rate of substance abuse at least once was $1.3 \%$. Illegal substance abuse rates are significantly on the rise in our country, as is the case in the world. The starting age for substance abuse is gradually dropping as well (17 \pm 2 years) [1].

The results of our study revealed that the mean age of the substance abusing group was 25 years of age, while the youngest participant's age was 17. Since the average age for substance abuse was $17 \pm 2$ both in our country and the world, as was revealed by the review of data during the planning stage of our study, healthy and young individuals representing a similar age group were selected for the control group. We believe, however, that the mean age of group I was higher because the participants in this group were those who had been substance abusers for an average of 5 years and had decided to quit. Moreover, the participants of group II were of a homogeneous group; that is to say, the group was formed by participants of similar ages who had presented to the health center with the objective to enroll at a program in college, while group I was composed of individuals of varying ages from 17 to 39 . This fact contributes to the significant age difference between the two groups.

Although the participants were selected for the study regardless of sex, the results of our study revealed that both the control group and the study group were coincidentally made up of only male individuals. It can be argued that the fact that all the participants were male can be explained as a result of the fact that the male population abuses substances more than the female population, both in our country and worldwide.
Newly developed synthetic substances and the emergence of alternative modes of abuse of such well-known substances as heroin and cocaine also contribute to the problem of popularization of substance abuse. Especially the common view that substance abuse by inhalation was less harmful than the IV mode among substance abusers has recently led to an increase in substance abuse through inhalation $[11,12]$. The results of our study showed that both heroin and cocaine were most commonly abused through inhalation and the rate of this mode of abuse was significantly higher than that of the IV mode. There were 25 (62.5\%) individuals who had only used the inhalation mode, 13 (32.5\%) who had used the inhalation mode together with the IV mode, and 2 (5\%) who had only used the IV mode in our study. The data collected as a result of our study supported the results of studies conducted on the evaluation of modes of substance abuse in our country and Europe. Studies in the literature have reported that the rate of substance abuse by the IV mode dropped from $43 \%$ to $35 \%$ between 2003 and 2006, while the rate of the inhalation mode was $65 \%$ in 2006 . It has also been reported that approximately half of cocaine abusers (55\%) inhaled the substance through their noses, almost one third (32\%) inhaled the substance by smoking it with tobacco, and a smaller group (9\%) injected it [1].

While respiratory system symptoms such as shortness of breath, coughing, phlegm, chest pain, and hemoptysis were addressed within the scope of our study, the symptoms that immediately followed substance abuse were specifically considered significant. All the five symptoms which were investigated were seen at a significantly 
higher rate in group I. The most commonly detected symptom was shortness of breath (70\%). This was followed by coughing (50\%), phlegm (47.5\%), chest pain (32.5\%), and hemoptysis (22.5\%). We are of the opinion that the data we collected can be considered to be a sign of the damage that substance abuse inflicts on the respiratory system and can initiate substance addicts to present to hospitals earlier with respiratory complaints, as was our aim at the onset of our study.

The fact that the participants in group I had been smoking and abusing other narcotic substances together raises the question whether smoking constitutes the first step of substance addiction. All the members of group I had more than 10 pack-years of smoking history. The mean rate of smoking was found to be 13.8 pack-years in group I, while it was 3.89 pack-years in group II.

Thinking that a comparison of pack-years of smoking between the groups could be misleading, as the mean age of group I was higher, we compared the starting age of smoking between the groups and found that the participants in group I had started smoking at earlier ages. It was found that the median figure for the starting age of smoking in group I was 13 years of age, while it was 18 years of age in group II. If we consider the fact that the mean age of group I was 25, we can state that heavy smoking at early ages is observed among substance abusers. These differences mau also be related to the fact that group I was composed of individuals coming from a wider array of groups with different socio-cultural and educational levels, while group II was composed of young individuals about to attend college, all of whom were high-school graduates with prospective majors which necessitated bodily coordination such as firefighting and physical education.

The respiratory system examination results of the members of group I revealed that they had rhonchus, which is one of the pathological respiratory sounds, at a statistically significant frequency. Rhonchus was more frequently heard especially in patients who had been evaluated within the first week of their latest substance abuse. Rhonchi can be considered to be a clinical reflection of bronchospasm related to substance inhalation. Studies in the literature support this idea as well. Dose-dependent acute bronchospasm and an asthmatic crisis-like picture can be seen as a result of high-dose inhalation of heroin by healthy individuals without any asthma diagnosis. Heroin gives way to acute bronchospasm related to histamine secretion by inducing an increase in mast cell degranulation. Bronchospasm can frequently progress to serious and fatal levels [13-16].

Within the scope of our study, a total of 6 patients in group I had pathological signs as revealed by their PA pulmonary graphs, and 2 out of these
6 had calcified pulmonary nodules, 1 had millimetric pulmonary nodules, 1 had pleural thickening, and 2 had atelectatic band formation. Tuberculosis was not found in any participant. The relationship between the participants' pulmonary graph results and the substances they abused could not be evaluated since they had abused substances in multiple combinations and through both IV and inhalation modes. Studies in the literature, however, have reported that pathological symptoms, which we also detected, were seen in individuals who abused heroin, cocaine, and marijuana both separately and together $[14,17]$.

When the FEV 1 and FVC values, which are among spirometric parameters, were compared within the scope of our study, no significant difference was found between the two groups, while the $\mathrm{FEV}_{1} / \mathrm{FVC}$ parameter was significantly lower in group I. The mean $\mathrm{FEV}_{1} / \mathrm{FVC}$ value in group I was 86 , while it was 94 in group II. It was observed that the mean $\mathrm{FEV}_{1} / \mathrm{FVC}$ values were not at levels considered to be obstructive in spite of this statistically significant low value. However, the fact that group I had lower $\mathrm{FEV}_{1} / \mathrm{FVC}$ values can be interpreted as a spirometric reflection of the high rhonchus level found in this group as revealed by physical examination. Studies in the literature have reported that especially marijuana did not lead to a change in $\mathrm{FEV}_{1}$ while it caused an increase in FVC and did not change or gave way to a decrease in the $\mathrm{FEV}_{1} / \mathrm{FVC}$ ratio [18]. The facts, however, that $\mathrm{FEV}_{1} / \mathrm{FVC}$ is a spirometric datum which decreases gradually by age and the mean age of group I was found to be older and also history of smoking and the higher probability of obstructive pulmonary diseases related to smoking in group I should be taken into consideration during evaluations.

Significant differences were found between group I and group II with regards to all the parameters recorded as vital signs. Substance abusers had higher heart rates, lower oxygen saturation, and higher respiratory rates. We also thought that the data collected could be related to withdrawal symptoms as the patients had not been abusing substances for at least a day although it was known that tachycardia could be seen as cocaine abuse had a sympathomimetic effect $[13,17]$.

Basically, the part of SGRQ which has been used to evaluate the symptoms of COPD during diagnosis and treatment, which was developed in order to evaluate the treatment used, was not asked to any participant. Therefore, there was a difference in scoring compared to standard scoring. It was, however, possible to achieve a healthy evaluation as the test was conducted in the same way with each participant [10]. When the scores obtained were compared, it was seen that the respiratory symptom scoring in group I was significantly high. It was noted that substance abusers had higher 
rates of respiratory system complaints than those of healthy individuals although they were not particularly aware of these complaints in their everyday lives.

In conclusion, the results of our study revealed that substance addicts most frequently preferred the inhalation mode when abusing narcotic substances. The number of individuals who abused more than one narcotic substance was striking. Moreover, it was also determined that they were heavily smoking besides narcotic substance abuse. Respiratory symptoms and bronchospasm signs as revealed by physical examination were significantly higher in this group. Although no comparisons could be drawn as the number of participants who had abused substances through the IV mode was small, we believe that these results could be related to the fact that they had abused addictive substances through the inhalation mode as well.

We predict that these cases with respiratory complaints will frequently be met in the daily practices of physicians in the future, having been affected by the gradual popularization of narcotic substance abuse through the inhalation mode. We also believe that efforts to prevent smoking addiction in society can be developed so as to include narcotic substance abuse, especially by young individuals, which in turn can contribute to a decrease in future risks.

\section{Conflict of interest}

The authors declare no conflict of interest.

\section{References}

1. The Grand National Assembly Of Turkey The Parliamentary Inquiry Commission Established To Search For Problems Regarding Substances, Specifically Drugs, Dependence, Drug Trafficking And To Discern The Necessary Precautions. Summary Of The Parliamentary Inquiry Commission Report, 2009, Ankara, Turkey.

2. Ertekin G, Çakmak D. A study on substance abuse culture. J Depend 2001; 2: 16-20.

3. Aveyard P, Markham WA, Lancashire E, et al. The influence of school culture on smoking among pupils'. Soc Sci Med 2004; 58: 1767-80.

4. Kuntsche E, Jordan M. Adolescent alcohol and cannabis use in relation to peer and school factors. Drug Alcohol Depend 2006; 84: 167-74.

5. Nguyen ET, Silva Cl, Souza CA, Müller NL. Pulmonary complications of illict drug use: differential diagnosis based on CT findings. J Thorac Imaging 2007; 22: 199206.

6. Megarbane B, Chevillard L. The large spectrum of pulmonary complications following illict drug use: features and mechanisms. Chem Biol Interact 2013; 206: 444-51.

7. Benson MK, Bentley AM. Lung disease induced by drug addiction. Thorax 1995; 50: 1125-7.

8. Miller MR, Hankinson J, Brusasco V, et al. Standardisation of spirometry. Eur Respir J 2005; 26: 319-38.
9. Macintyre N, Crapo RO, Viegi G, et al. Standardisation of the single-breath determination of carbon monoxide uptake in the lung. Eur Respir J 2005; 26: 720-35.

10. Paul J. St George's Respiratory Questionnarre Manual, Version 2.3. 2009

11. Caponnetto P, Auditore R, Russo C, et al. Dangerous relationships: asthma and substance abuse. J Addict Dis 2013; 32: 158-67.

12. Whale $\mathrm{Cl}$, Molyneux AW, Ward MJ. Inhaled heroin causing a life-threatening asthma exacerbation and marked peripheral eosinophilia. Br J Hosp Med 2007; 68: 332-3.

13. Wolff AJ, O'Donnell AE. Pulmonary effects of illicit drug use. Clin Chest Med 2004; 25: 203-16.

14. Gottlieb LS, Boylen TC. Pulmonary complications of drug abuse. West J Med 1974; 120: 8-16.

15. Koçoğlu H, Katı I, Demirel CB, Abbasov Ü. Pulmonary edema due to heroin intoxication: a case report. Van Med J 2000; 7: 40-2.

16. Sporer KA, Dorn E. Heroin-related noncardiogenic pulmonary edema. Chest 2001; 120: 1628-32.

17. Goway MB, Marder SR, Hanks DK, et al. Thoracic complications of illict drug use: an organ system approach. Radiographics 2002; 22: 119-35.

18. Lee MHS, Hancox RJ. Effects of smoking cannabis on lung function. Expert Rev Respir Med 2011; 5: 537-47. 